\title{
Tensiones entre sindicalismo y feminismos en un periodo de demandas y reivindicaciones para la transformación social
}

\section{Tensions between Trade Unionism and Feminisms in a Period of Requests and Modifications for Social Transformation}

\section{Florencia Laura Rovetto}

Universidad Nacional de Rosario, Argentina. Doctora en Periodismos y Ciencias de la Comunicación por la Universidad Autónoma de Barcelona.

florencia.rovetto@gmail.com | https://orcid.org/0000-0002-8900-5363

\section{Carla Susana Millán}

Comisión Ejecutiva de la Central de Trabajadores de la Argentina (CTA-T), regional Rosario, Argentina Licenciada en Ciencia Política por la Universidad Nacional de Rosario. carlamarchisin@gmail.com | https://orcid.org/0000-0001-6608-5471

Recibido: 25 de julio de 2019. Aprobado: 9 de diciembre de 2019

DOI: $10.25100 /$ lamanzanadeladiscordia.v14i2.8771

Artículo de investigación

Financiación o proveniencia del artículo: Este artículo es resultado de las actividades de formación e investigación en torno al problema de la división sexual del trabajo desarrolladas en el marco del Centro de

Investigaciones Feministas y Estudios de Género de la Facultad de Ciencia Política y Relaciones

Internacionales (CIFEG) de la Universidad Nacional de Rosario, y del trabajo de investigación titulado:

"Sindicalismo y género: una historización de la Central de Trabajadores de la Argentina".

¿Cómo citar este artículo? / How to quote this article?

Rovetto, F. L., y Millán, C. S. (2019). Tensiones entre sindicalismo y feminismos en un periodo de demandas y reivindicaciones para la transformación social. La Manzana de la Discordia, 14(2), 5-27. doi:

10.25100/lamanzanadeladiscordia.v14i2.8771 


\title{
Resumen
}

En este artículo se analiza la experiencia de la Central de Trabajadores de la Argentina (CTA) desde sus orígenes (1991) hasta su fractura (2010), tomando aportes de los estudios de género para analizar la incorporación de las demandas y reivindicaciones de las trabajadoras como sujetos políticos en los espacios sindicales, atendiendo a tres aspectos centrales: a) la articulación entre la central sindical y el movimiento de mujeres y feminista, b) la transversalización de la perspectiva de género en sus principales políticas, y c) la participación de las mujeres en las instancias de conducción nacional. Para ello nos valemos de diferentes fuentes bibliográficas, documentales y entrevistas a las dos titulares de la Secretaría de Género e Igualdad de Oportunidades, para recuperar sus miradas acerca de estas cuestiones problemáticas en un contexto atravesado por las tensiones y disputas que confrontan el protagonismo político de los feminismos con las políticas de restauración neoliberal y neopatriarcal, tanto en el país como a nivel global. Del análisis de la experiencia de la CTA se puede ver una propuesta innovadora frente de modelo sindical tradicional con el acento puesto en colectivos sociales emergentes, incorporando, paulatinamente, las causas impulsadas por los movimientos feministas.

Palabras clave: género; feminismo; sindicalismo; CTA; equidad.

\begin{abstract}
This article analyzes the experience of the Central of Workers of Argentina (CTA) from its origins (1991) to its fracture (2010), taking contributions from gender studies to analyze the incorporation of the demands and demands of the workers as subjects politicians in the union spaces, attending to three central aspects: a) the articulation between the trade union center and the women's and feminist movement, $b$ ) the mainstreaming of the gender perspective in its main policies and c) the participation of women in the national driving instances. To do this we use different bibliographical and documentary sources, as well as interviews with the two heads of the Gender and Equality of Opportunities Secretariat of the CTA to recover their views on these problematic issues in a context crossed by the tensions and disputes that They confront the political protagonism of feminisms with neoliberal and neopatriarcal restoration policies, both in the country and globally.
\end{abstract}

Keywords: gender; feminism; unionism; CTA; equity. 


\section{Presentación del caso y claves interpretativas}

La Central de Trabajadores de la Argentina (en adelante CTA) se originó como respuesta a la necesidad de construir una alternativa sindical a la Confederación General del Trabajo (en adelante CGT) ${ }^{1}$ que enfrentara las políticas neoliberales llevadas adelante en la década de 1990 por el gobierno de Carlos Menem. Nació en el año 1991 como encuentro de organizaciones, para convertirse, en 1992, en Congreso de Trabajadores Argentinos, hasta transformarse en Central Sindical en el año 1996.

Durante la década de 1990, logró consolidarse como actor político, combinando nuevos repertorios de acción que movilizaron a la ciudadanía con las herramientas clásicas del sindicalismo. En la siguiente década, durante los dos primeros mandatos presidenciales kirchneristas ${ }^{2}$, pretendió articular demandas desde propuestas políticas globales cuando hubo mayor protagonismo de los conflictos laborales por recomposición salarial y se activaron las negociaciones colectivas encabezadas por los sindicatos tradicionales (Sénen González, 2013); sin embargo, enfrentó las dificultades que implicaba ser una central con poca incidencia en el sector industrial. También en esos años, las diferentes lecturas y posicionamientos frente al proceso político argentino, así como el debate no sintetizado sobre la herramienta política de las personas trabajadoras generaron tensiones internas por disputas estratégicas a desarrollar. Todos estos elementos se combinaron de distintas maneras para finalizar con la fractura de este espacio en el año 2010, cuando se conformaron la CTA-Autónoma, por un lado, y la CTA de los trabajadores, por el otro.

Así pues, surgida en la década de 1990 a partir de las condiciones que generaron las reformas neoliberales en curso, se constituyó en una de las novedades más importantes del campo sindical por varias cuestiones: 1) vertebrar la necesidad de "refundar el sindicalismo"; 2) promover la autonomía sindical respecto del Estado, los partidos políticos y los grupos económicos; 3) fortalecer la democracia sindical; y 4) incluir a organizaciones sociales que expresaban las demandas de los sectores populares (Armelino, 2004).

La propuesta también fue innovadora por incorporar el mecanismo de afiliación directa, incluyendo, además de sindicatos, principalmente, del sector estatal entre los que se destacan la Asociación de trabajadores del Estado (ATE) y la Confederación de Trabajadores de la Educación de la República Argentina (CTERA), otro tipo de asociaciones como las organizaciones sociales emergentes. Entre estas últimas, se

\footnotetext{
${ }^{1}$ La CGT es la central sindical histórica de Argentina fundada en 1930 como consecuencia de un acuerdo entre socialistas, sindicalistas revolucionarios, comunistas e independientes para generar una central sindical unitaria y plural. En general tuvo mayoría socialista hasta 1945 y peronista desde entonces. Actualmente es la central mayoritaria e internacionalmente está afiliada a la Confederación Sindical Internacional (mundial), a la Confederación Sindical de las Américas y a la CCSCS (Mercosur).

${ }^{2}$ El kirchnerismo es un movimiento político de raíz peronista, surgido en el año 2003, que reúne los principales postulados ideológicos plasmados en los gobiernos de los presidentes Néstor Kirchner (20032007) y Cristina Fernández de Kirchner (2007-2015), los que, en conjunto, ocuparon el Poder Ejecutivo de Argentina durante 12 años.
} 
encuentran la Federación Nacional de Trabajadores por la Tierra, la Vivienda y el Hábitat (FTV) y la Asociación de Meretrices de la República Argentina (AMMAR), entre otras.

Otra novedad aportada por la CTA es que varios de los sindicatos adheridos y la propia central, incorporaron intelectuales, investigadores sociales y profesionales, nucleándolos en centros de investigación, cuerpos docentes para la formación profesional y sindical, grupos de análisis sobre la realidad argentina contemporánea, etc. (Bonaccorsi y Carrario, 2012, p. 133).

Estas características iniciales permitieron extender su ámbito de representatividad más allá del laboral, posibilitando la incorporación de movimientos sociales diversos, vinculados a las organizaciones barriales y de personas desocupadas, así como a otros activistas, trabajadoras que no se encontraban sindicalizadas ni organizadas colectivamente.

Uno de los datos destacados en este período, en el que se profundizó la reestructuración del mundo del trabajo ${ }^{3}$, fue el aumento de la participación de las mujeres. Si bien la incorporación de las mujeres al mercado laboral argentino se desarrolló con mayor intensidad a partir de la década de 1970 y avanzó gradualmente en las décadas subsiguientes, Aspiazu (2014) señala que la década de 1990 implicó una aceleración de ese proceso con un fuerte auge en la crisis de 2001/2002 $2^{4}$

A principios de este siglo, el profundo incremento del desempleo, la subocupación y el deterioro de los salarios generaron la necesidad de que muchas mujeres disputaran un espacio en el mercado, para convertirse en el principal ingreso o complementar los ingresos del hogar (Pautassi, 1998). Durante este período, según datos extraídos de la Encuesta Permanente de Hogares del Instituto Nacional de Estadísticas y Censos (INDEC) "la tasa de actividad de las mujeres pasó del 30\% al 35\% y, una vez superada la crisis, se mantuvo en valores cercanos al 40\% hasta la actualidad" (Aspiazu, 2014, p. 16).

A pesar del significativo aumento de las mujeres en el mercado de trabajo, su incorporación fue y continúa siendo asimétrica respecto de los varones. La concentración en ciertos oficios, la vulnerabilidad laboral y la brecha salarial son algunas de las características de

\footnotetext{
${ }^{3}$ Al respecto, Basualdo (2003) describe los cambios que se producen durante esta década en cuanto a las normas sobre la determinación salarial, la limitación del derecho a huelga, la alteración del régimen de vacaciones, la privatización del sistema de previsión de accidentes de trabajo, la puesta en vigencia de diversas formas de contratos temporarios que disminuyen el costo para los empresarios, la reducción de los aportes patronales a la seguridad social y las asignaciones familiares, disminución de las indemnizaciones por despidos, etc. Todos estos factores que propician una notable precariedad en el mercado de trabajo y una exaración de las condiciones de explotación.

${ }^{4}$ La crisis de diciembre de 2001 en Argentina fue una crisis política, económica, social e institucional, potenciada por una revuelta popular generalizada bajo el lema "iQue se vayan todos!", que causó la renuncia del entonces presidente, Fernando de la Rúa, dando lugar a un período de inestabilidad política durante el cual cinco funcionarios ejercieron la Presidencia de la Nación. Más información al respecto de las causas de esta crisis y sus repercusiones en Castellani y Schorr (2003) y Cortés Conde (2003).
} 
este acceso desigual. Las principales explicaciones de este fenómeno remiten a lo que la teoría feminista ha definido como división sexual del trabajo, que se basa en la delimitación de las dos esferas, opuestas en términos de jerarquías, con las que se organiza la vida social: la privada, infravalorada, asociada a las mujeres y vinculada al trabajo reproductivo y doméstico, y la pública, sobrevalorada, asociada a los varones, relacionada con la participación en los ámbitos del trabajo productivo y los espacios públicos (Pateman, 1995).

La división sexual del trabajo también se expresa mediante mecanismos como la segregación laboral horizontal y vertical, el techo de cristal o la brecha salarial ${ }^{5}$, en tanto mecanismos de organización sociolaboral solapados o explícitos. Dichos mecanismos fraguan cotidianamente "las relaciones sociales de sexos, comprendidas como relaciones desiguales, jerarquizadas, asimétricas o antagónicas de explotación entre dos categorías de sexos construidas socialmente" (Hirata, 1997, p. 54).

Se sostiene que el modo de inserción para las mujeres continúa siendo desigual, sustentado en una segregación horizontal por rama de actividad y una vertical por jerarquía (Rojo Brizuela y Tumini, 2008). A su vez, los estereotipos de género que asocian a las mujeres con determinadas características se trasladan al mercado de trabajo en virtud de una mayor participación en áreas "blandas", de servicios, de cuidados o vinculadas a tareas reproductivas. Así también, los atributos valorados negativamente son una fuente de limitación a la hora de acceder a cargos de jerarquía que implican atributos menos "empáticos" o de jefatura y control asociados a los varones. Esta barrera invisible que opera en la posibilidad de las mujeres de ocupar lugares de mando fue denominada techo de cristal $^{6}$, porque limita material y subjetivamente su ascenso laboral a los cargos ejecutivos

\footnotetext{
${ }^{5}$ Según un informe del CEPA, la brecha de ingresos entre varones y mujeres se mantiene entre el 20 y $30 \%$ desde hace más de una década. La brecha salarial consiste en un indicador económico y socio-ocupacional complejo que no se reduce al incumplimiento del derecho constitucional "igual remuneración por igual tarea", sino que resulta de la comparación de masas salariales (cantidad de horas por salario). Por ejemplo, en Argentina la tasa de actividad femenina al primer trimestre de 2018 ascendió a 48,5\% mientras que la de los varones registró $69,5 \%$. La significativa diferencia entre ambas es del 20\%. Además del diferencial en horas trabajadas, la segregación ocupacional se considera por la división entre actividades feminizadas y actividades masculinizadas, donde las primeras perciben menores ingresos. La inserción ocupacional de gran parte de las mujeres tiene lugar en actividades consideradas feminizadas, asociadas a tareas del cuidado. Asimismo, en la ponderación de la brecha salarial se debe incluir que las mujeres padecen una doble jornada laboral (se estima que destinan seis horas promedio por día a tareas domésticas), pero solo perciben ingresos por una jornada, la cual tiene - por otro lado- menos horas que la de los varones y por ende se traduce en la percepción de ingresos menores (Strada, 2018).

${ }^{6} \mathrm{Si}$ bien, en los últimos años, la metáfora del techo de cristal ha tenido amplia difusión y adhesión para explicar los límites no escritos o invisibles en el avance social y político de las mujeres (López Diez, 2002), algunas autoras han procurado revisar este concepto, sosteniendo que se parte del error al pensar que mujeres y varones tienen idénticas posiciones de partida y que la metáfora singulariza uno de los obstáculos visibilizando otras dificultades que encuentran a lo largo de su vida laboral. Así, Eagly y Carli (2007) sostienen que más que una barrera con la que chocan y que frena en seco su ascenso profesional, se trata de un laberinto lleno de obstáculos del que es muy complejo salir.
} 
tanto en las organizaciones laborales como en los espacios asociativos gremiales y sindicales.

Este fenómeno también tiene su correlato en la afiliación sindical femenina. En los sindicatos de trabajadoras docentes y estatales, gremios mayoritarios en la composición de la CTA, los números indican que para el año 2011, luego de 20 años de su creación, las mujeres configuraban el $75 \%$ de las personas afiliadas en los gremios de la enseñanza, el $55 \%$ en los sindicatos del sector salud y el $46 \%$ en la administración pública. Estos porcentajes de afiliación se corresponden con la distribución por sexo del empleo en estas ramas de actividad, que son las de mayor tasa de feminización. Sin embargo, si ponemos el foco en los puestos de dirección sindical, observamos que la simetría de la relación se altera completamente con una fuerte primacía masculina (Aspiazu, 2015, p. 16).

Por otra parte, desde el año 2002, Argentina cuenta con la Ley de cupo sindical femenino como medida de acción positiva para favorecer a las mujeres en lugares de representación, no solo en términos de cantidad de cargos ocupados por varones y mujeres sino de las funciones y relevancia de los mismos dentro de la organización (Senado y Cámara de Diputados de la Nación Argentina, 2002) ${ }^{7}$. Cierto es que todos los procesos y sistemas de negociación colectiva constituyen instrumentos fundamentales para la consecución de la equidad de género y la igualdad de oportunidades en el mundo del trabajo, y ello tanto en los ámbitos de acceso al trabajo, como en cuanto a la capacitación y la estabilidad en el empleo. Sin embargo, a casi dos décadas de la sanción de esta ley, en el presente solo 4 sindicatos la cumplen, tal como indicaran Bonaccorsi y Carrario (2012):

producto de negociaciones políticas y reclamos desde distintos espacios de mujeres realizados durante la década del 90, se produce un clivaje en la participación de las mujeres en la estructura sindical argentina. Con la promulgación de la Ley $\mathrm{N}^{\circ} 25.674$, el sistema de cuotas se impone para asegurar que las candidaturas de mujeres a los órganos de dirección incluyan un número proporcional a la afiliación. Sin embargo, desde su implementación, la Ley de Cupo Sindical Femenino, ha tenido altibajos en cuanto a la incorporación de mujeres en sus estructuras de decisión, que deducimos es producto de una resistencia patriarcal (p. 127).

Además, según un reciente informe elaborado por el Ministerio de Trabajo de la Nación, las mujeres están a cargo del $18 \%$ de secretarías, subsecretarías o prosecretarías de los

\footnotetext{
${ }^{7}$ La Ley No 25.674 denominada de “Cupo Femenino Sindical” modificó el art. 18 de la Ley No 23.551 (Ley de Asociaciones Sindicales) El nuevo cuerpo jurídico establece la integración obligatoria de mujeres en cargos electivos y representativos de las organizaciones sindicales, determinando que el porcentaje femenino será de un mínimo de un $30 \%$ cuando el número de mujeres alcance o supere ese porcentual sobre el total de los trabajadores y trabajadoras.
} 
sindicatos, de las cuales, el $74 \%$ corresponden a áreas de igualdad, género o servicios sociales (Presidencia de la Nación, Ministerio de trabajo, empleo y seguridad social, s.f.) ${ }^{8}$.

Por último, interesa hacer referencia a los objetivos de la transversalización de la perspectiva de género en la CTA, tanto en las actividades de la organización (existencia de comisiones específicas o inclusión de cláusulas en los estatutos), como en la inclusión de la dimensión de género y sus implicancias en todos los temas abordados, entendiendo que debe tratarse como una dimensión transversal y no una cuestión aislada que requiere solamente de políticas focalizadas en las mujeres (Aspiazu, 2015).

Considerando estos elementos, a continuación, se propone reflexionar sobre la articulación de la CTA con el movimiento de mujeres y feminista a nivel nacional que, en paralelo, ha tenido un crecimiento exponencial en las últimas décadas en nuestro país (Burton, 2013). Para ello se pregunta por la presencia de las mujeres como sujetos políticos en la CTA a lo largo de los 19 años que transcurrieron entre sus orígenes en 1991 hasta su ruptura en el 2010. Esta pregunta general permite situar otros interrogantes en torno a las articulaciones que se pueden identificar entre la central sindical y el movimiento de mujeres y feminista desde su creación hasta su ruptura. ¿En qué medida la perspectiva de género se manifestó como eje de la política sindical en la CTA? ¿Cómo fue la participación de las mujeres en las instancias de decisión de la CTA a nivel nacional?

Para ello, en los siguientes apartados se analiza, en primer lugar, la composición de las distintas Comisiones Ejecutivas Nacionales (en adelante CEN), relevando la cantidad de mujeres que participaron, su función y permanencia en dicha instancia de conducción, durante todo el período seleccionado para este estudio, valiéndonos de la información contenida en archivos de la CTA sobre este espacio de dirección para los períodos 19921995; 1995-1997; 1997-2000; 2000-2006 y 2006-2009. En segundo lugar, se indaga sobre la transversalización de la perspectiva de género en el período estudiado, relevando los documentos elaborados por la propia Central y a partir de las entrevistas realizadas a dos secretarias de género: Mabel Gabarra y Estela Díaz, que se sucedieron en ese cargo entre los años 2000 y $2006^{9}$. Por último, interesa reflexionar sobre la articulación entre las políticas de género de la CTA y el programa actual de reivindicaciones y luchas del movimiento de mujeres y feminista argentino en un contexto caracterizado por grandes movilizaciones y la visibilidad de demandas, tomando las opiniones relevadas en las entrevistas realizadas.

\footnotetext{
8 El informe fue difundido por el Ministerio de Trabajo en el marco del 8 de marzo de 2018 ("Día Internacional de la Mujer Trabajadora") que implicó una jornada de huelga protagonizada por trabajadoras y organizaciones feministas. Cabe aclarar que, desde septiembre del mismo año, el Ministerio de Trabajo pasó a tener rango de Secretaría, para dejarlo con funciones reducidas y subordinado al Ministerio de Producción.

${ }^{9} \mathrm{La}$ intención para este trabajo, que es una primera aproximación al tema, fue entrevistar a las tres Secretarias de Género e Igualdad de Oportunidades que tuvo la CTA a nivel nacional durante el período que abarca esta investigación, aunque solo se consiguió hacerlo con dos de ellas. De todas formas, sus aportes fueron sustanciales respecto de los objetivos que se proponen aquí.
} 


\section{Presencia de mujeres y enfoque de género en la CTA}

En este apartado se procura describir y analizar la participación de las mujeres en la instancia de conducción nacional de la CTA; establecer en qué medida la perspectiva de género se manifestó como eje de la política sindical e identificar las articulaciones entre la CTA y el movimiento de mujeres y feminista.

Tal como se mencionó anteriormente, para este análisis se recopilaron datos sobre la composición de la primera Mesa Nacional del CTA y las sucesivas Comisiones Ejecutivas Nacionales, obtenida en la Biblioteca de la Central a partir de fuentes secundarias como anuarios, actas de asunción de autoridades y actas de proclamación de la Junta Electoral Nacional. Por otra parte, la recolección de documentos, volantes, declaraciones y posicionamientos públicos de la CTA para algunos hitos de su historia, permitió analizar la inclusión de la perspectiva de género como eje de la política sindical, atendiendo al uso del lenguaje, las categorías de análisis propuestas y los actores a convocar para el despliegue de sus políticas ${ }^{10}$. Por último, los testimonios relevados en las entrevistas realizadas a quienes ocuparon la Secretaría de Género e Igualdad de Oportunidades durante el periodo analizado $^{11}$, constituyeron aportes de gran relevancia.

Las entrevistas fueron realizadas entre los meses de mayo y junio de 2017. Los ejes de indagación versaron sobre: acontecimientos relevantes vinculado a la participación de las mujeres en la historia de la CTA; el diálogo entre sindicalismo y feminismo, así como las articulaciones entre ambos movimientos; el proceso previo a la institucionalización de la Secretaría de Género y la conformación de espacios de mujeres; la agenda de género en la agenda de la CTA; y la participación de las mujeres en las instancias de conducción.

\section{Participación de las mujeres: comisiones ejecutivas, cupo sindical y secretarías}

Este apartado tiene como objetivo recuperar una de las políticas de acción positiva que implementó la Central, el cupo femenino, así como sus potencialidades en relación a la incorporación de un número mayor de mujeres a las instancias de conducción, y sus limitaciones, en tanto roles y permanencia en las secretarías. Para esto se presenta el análisis y los resultados de los cargos ocupados por mujeres y varones, en términos porcentuales, en una de las instancias de conducción nacional de la CTA para el período 1992-2009. También se describe la permanencia de las mujeres en los mismos, así como el tipo de cargos a los que accedieron.

\footnotetext{
${ }^{10}$ Es importante mencionar que el acceso a datos fue una tarea compleja debido a la escasa organización registral y sistematización archivística de los mismos por parte de la CTA.

${ }^{11}$ Durante el tiempo que abarca el análisis de esta investigación se sucedieron tres secretarias de género e igualdad de oportunidades en la CTA a nivel nacional: Mabel Gabarra estuvo en este cargo entre los años 2000 y 2003; Estela Díaz para el período 2003-2006 y Alejandra Angriman entre los años 2006 y 2009. Solo las primeras dos accedieron a ser entrevistadas para esta investigación.
} 
Es importante mencionar que en el mes de junio de 2000 se reformó el estatuto de la CTA que incorporó la Secretaría de Género e Igualdad de Oportunidades y estableció un cupo femenino del 20\% para los cargos de conducción (Retamozo y Morris, 2015), dos años antes de la Sanción de la Ley de Cupo Sindical Femenino. También se quiere remarcar que, como antecedente a la institucionalización de esta secretaría y a la iniciativa del cupo, existieron espacios de mujeres que fueron fundamentales para que esto se implementara, tal como lo relata Estela Díaz ${ }^{12}$ :

Previo a tener la institucionalidad de la secretaría, en la CTA y en los sindicatos, funcionaban áreas, direcciones y espacios encuentros para las mujeres. Con el tiempo, estos espacios se han ido consolidando como parte de la conducción en cada organización (...), en el inicio de la CTA hubo una participación importante de mujeres feministas de diversas organizaciones sindicales y sociales. Eso posibilitó que se discutiera la creación de la secretaría y la agenda de género como programa de acción. Recordemos que este proceso se dio en el marco de la Conferencia Mundial de la Mujer de Beijing (1995). Muchas sindicalistas fueron a la Conferencia y las que no fueron, como mi caso, participamos de los debates previos y posteriores. Fue un momento de mucha reflexión en torno a estas temáticas, precedida incluso por las discusiones que nos habíamos dado en el proceso de la reforma constitucional del 94 (como el cupo femenino, por ejemplo). También momentos de grandes discusiones legislativas en temas como violencia familiar, ley de salud sexual, etc. (Estela Díaz, entrevista personal, 06 de junio, 2017)

Estos espacios de encuentro y sus agendas de debate existieron en zonas grises dentro de los marcos institucionales de la Central. A continuación, en la Tabla 1, se identifican los porcentajes de participación y su impacto en la misma.

Tabla 1. Porcentajes de participación de las mujeres y los varones en las CEN.

Período 1992-2010 13

\begin{tabular}{cccccc}
\hline Período CEN & Varones & Mujeres & $\% \mathbf{~ V}$ & $\% \mathbf{M}$ & Total \\
\hline $\mathbf{1 9 9 2 - 1 9 9 5}$ & 11 & 1 & $91,7 \%$ & $8,3 \%$ & 12
\end{tabular}

12 Estela Díaz es actualmente la secretaria de género e igualdad de oportunidades de la CTA de los Trabajadores (luego de la fractura en 2010, la CTA se dividió en dos. Por un lado, se conformó la CTAAutónoma y por otro, la CTA de los Trabajadores). Es Técnica Superior en Programación y durante sus años de estudiante de Letras en la Universidad de la Plata inició su militancia política. Es referente de la Federación de Tierra y Vivienda y Hábitat, integrante de la Campaña Nacional por el Derecho al Aborto Legal, Seguro y Gratuito y dirige el Centro de Estudios: mujeres y trabajos de la Argentina (CEMyET- CTA), militante social, política y feminista.

${ }^{13}$ No incluye Vocalías ni Comisión Revisora de Cuentas por no estar disponible esta información para todo el período. Dentro de la columna "Período CEN" se incluye, para el lapso 1991-1995 la información sobre la Mesa de Conducción Nacional por no existir aún la CEN. 


\begin{tabular}{cccccc}
$\mathbf{1 9 9 5 - 1 9 9 7}$ & 12 & 3 & $80 \%$ & $20 \%$ & 15 \\
$\mathbf{1 9 9 7 - 2 0 0 0}$ & 14 & 1 & $93 \%$ & $7 \%$ & 15 \\
$\mathbf{2 0 0 0 - 2 0 0 3}$ & 13 & 3 & $81 \%$ & $19 \%$ & 16 \\
$\mathbf{2 0 0 3 - 2 0 0 6}$ & 12 & 2 & $86 \%$ & $14 \%$ & 14 \\
$\mathbf{2 0 0 6 - 2 0 0 9}$ & 14 & 6 & $70 \%$ & $30 \%$ & 20 \\
Total & $\mathbf{7 6}$ & $\mathbf{1 6}$ & $\mathbf{8 3 \%}$ & $\mathbf{1 7 \%}$ & $\mathbf{9 2}$ \\
\hline
\end{tabular}

Fuente: elaboración propia con base en los datos aportados por la biblioteca de la CTA.

Los datos para el período arrojan que, de un total de 92 cargos, 76 fueron ocupados por varones y 16 por mujeres. Es decir, que desde el año 1992 hasta 2009 un 83\% de las secretarías fueron conducidas por varones y un $17 \%$ por mujeres. En este punto podemos considerar el notorio impacto de la Ley de cupo sindical femenino para el período 20062009, en donde por primera vez el porcentaje de mujeres en cargos de la CEN alcanzó el $30 \%$.

La información con la que se cuenta de la composición de la Primera Mesa Nacional del CTA para el año 1992, no proporciona la distinción por cargos, por lo cual quedará por fuera de este análisis.

Las secretarías que componen la CEN tienen distintas funciones de acuerdo al estatuto de la CTA. Para los fines de este análisis, se ha agrupado la participación de las mujeres de acuerdo a las secretarías en las que se desempeñaron y los fines perseguidos en las mismas: institucionales-gremiales-organizativos; administrativo-financieros; formativoscomunicacionales-acción social; nuevos derechos.

En el primer grupo están incluidas la Secretaría General, las dos Secretarías Adjuntas, la Secretaría Gremial, la Secretaría de Organización, la Secretaría de Relaciones Institucionales, la Secretaría de Relaciones Internacionales, y la Secretaría de Interior; en el segundo grupo se encuentran la Secretaría Administrativa, la Secretaría de Contabilidad y Finanzas, y la Secretaría de Actas; en el tercer grupo están la Secretaría de Comunicación y Difusión, la Secretaría de Asistencia Social, la Secretaría de Previsión Social, la Secretaría de Formación, Investigación, Proyectos y Estadísticas, la Secretaría de Condiciones y Medio Ambiente de Trabajo, y la Secretaría de Salud Laboral; y en el último grupo la Secretaría de Derechos Humanos, la Secretaría de la Juventud, y la Secretaría de Género e Igualdad de Oportunidades. 
Según los datos aportados en la Tabla 2, se observa un aumento en la participación de las mujeres en los grupos 2, 3 y 4, mientras disminuye en el grupo 1, llegando a cero para el trienio 2006-2009. Este período fue el único en el que la participación femenina llegó al $30 \%$, crecimiento que se produjo con la participación de una mujer en la Secretaría de actas (grupo 2) -que pasó de cero a una-; en la Secretaría de asistencia social, previsión social y formación (grupo 3) -pasando de cero a tres- y en la Secretaría de género e igualdad de oportunidades y juventud (grupo 4) -aumentando de uno a dos-, en comparación al período inmediato anterior. Es decir, en el momento de mayor incorporación de mujeres a esta instancia de dirección, desaparecen del grupo 1, vinculado a secretarías de representación institucional, gremial y organizativa y que ocupan los primeros lugares en las listas electorales, mientras crece su número en el resto de las secretarías ${ }^{14}$.

Tabla 2. Participación de mujeres en las secretarías de la CEN. Período 1995-2009

\begin{tabular}{|c|c|c|c|c|c|}
\hline $\begin{array}{l}\text { Período } \\
\text { Grupo }\end{array}$ & 1995-1997 & 1997-2000 & $2000-2003$ & $2003-2006$ & 2006-2009 \\
\hline $\begin{array}{l}\text { G.1 Actividades institucionales, } \\
\text { gremiales y organizativas }\end{array}$ & 2 & 1 & 1 & 1 & \\
\hline $\begin{array}{l}\text { G.2 Actividades administrativas } \\
\text { y financiera }\end{array}$ & & & & & 1 \\
\hline $\begin{array}{l}\text { G.3 Actividades formativas, } \\
\text { comunicacionales y de acción } \\
\text { social }\end{array}$ & 1 & & 1 & & 3 \\
\hline $\begin{array}{l}\text { G.4 Actividades vinculadas a } \\
\text { los nuevos derechos }\end{array}$ & & & 1 & 1 & 2 \\
\hline TOTAL & 3 & 1 & 3 & 2 & 6 \\
\hline
\end{tabular}

Fuente: elaboración propia en base a los datos aportados por la biblioteca de la CTA.

Los diez cargos ocupados por mujeres entre 1995 y 2009 son la Secretaría de Condiciones y Medio Ambiente de Trabajo, la Secretaría de Relaciones Internacionales, la Secretaría de Asistencia Social, la Secretaría de Previsión Social, la Secretaría de Actas, la Secretaría de Comunicación y Difusión, la Secretaría de Juventud y la Secretaría de Formación, Investigación, Proyectos y Estadística, siendo las Secretaría General Adjunta y Secretaría de Género e Igualdad de Oportunidades, las únicas en las que hubo mujeres a cargo por más de un período eleccionario. De todas ellas, Marta Maffei fue la única mujer que ocupó una secretaría durante más de un período.

\footnotetext{
${ }^{14}$ Los datos disponibles en torno al procedimiento de selección de integrantes en las listas se pudieron reconstruir a partir de la entrevista realizada a Mabel Gabarra. En su experiencia, Gabarra advierte que "cuando los varones se meten a decidir sobre los espacios de las mujeres generalmente causan problemas entre las mujeres”, y señala que, al igual que otras, la Secretaría de Género se negocia y las reglas de la negociación no son feministas.
} 
Además del bajo porcentaje de mujeres en esta instancia de conducción, es llamativa la baja permanencia en los cargos asumidos por parte de ellas (a excepción de Marta Maffei como Secretaria General Adjunta), cuestión que supone una rotación de referencias femeninas y un límite a la hora de desarrollar políticas. También es importante destacar que ninguna mujer ocupó el cargo de secretaria general. Una posible explicación sobre la escasa perdurabilidad que tuvieron las mujeres en las CEN es la que brinda Mabel, recuperando su propia experiencia:

Yo te puedo decir esto, cuando los varones se meten a decidir sobre los espacios de las mujeres generalmente causan problemas entre las mujeres. Y a mí me pasó eso muchas veces, tanto en la entrada a la Secretaría como por la falta de apoyo de algunas compañeras. Afortunadamente, hoy recuperé la relación con todas, pero en esos años fue duro aceptar que las lógicas masculinas de rivalidad y competencia también nos separaban a nosotras en el espacio sindical. Y después, al salir me pasó exactamente lo mismo, porque yo promocioné e incluso hablé para que hubiera varias compañeras que pudieran asumir la conducción y después la terminaron negociando entre sectores. La Secretaría de Género en los sindicatos se negocia. Tuvimos la suerte de que fuera Estela Díaz, que es feminista, la que le diera continuidad, pero si hubiese asumido cualquier otra persona que no tenía idea era empezar de nuevo y tirar al diablo todo lo que yo había construido (Mabel Gabarra, entrevista personal, 20 de mayo, 2017).

Si bien este relato se refiere a una secretaría en particular, es ilustrativo respecto a la lógica que opera para conformar las listas y la distribución de roles en su interior; evidencia, además, que estos acuerdos se construyen en espacios donde las mujeres no están presentes porque están organizados en clave androcéntrica, lo cual supone dinámicas restrictivas para las dirigentes que acceden a estos espacios o para discutir las propias formas de funcionamiento (Gerez, 2018).

También es importante ponderar que la utilización del tiempo intensivo en la vida de ellas, debido a la sobrecarga que conlleva la conciliación entre la vida familiar, laboral y gremial, impacta en la calidad de vida de las mujeres y en la posibilidad de sostener una permanencia en los cargos de mayor responsabilidad (Cutuli y Martino, 2012; Rodríguez Enríquez, 2015). Este fenómeno contrasta si se observa la permanencia de los varones en lugares de conducción. A modo ilustrativo, solo basta mencionar los sucesivos mandatos de Víctor De Gennaro como secretario general de la CTA desde 1995 hasta 2006 (Gusmerotti, $2009)^{15}$.

\footnotetext{
15 Al observar la permanencia de los varones en las instancias de conducción, se puede referir que en el período 1995-2003, seis de ellos se mantuvieron en las mismas secretarías. Asimismo, si se incorporan los datos del último período (y se toma en conjunto el lapso de tiempo que va desde el año 1995 hasta 2006), en el cual se implementó el cupo sindical femenino del 30\%, se observa que cinco de ellos continúan con funciones en el secretariado.
} 
Hasta aquí se ha considerado relevante observar la cantidad de participantes mujeres, los cargos que ocuparon y la perdurabilidad en los mismos en la instancia de conducción nacional de la CTA; en el próximo apartado se analiza si su participación produjo cambios en los enfoques y en las políticas organizacionales tendientes a representar los intereses de este colectivo.

\section{Transversalización de la perspectiva de género en las políticas de la CTA}

La pregunta que guía esta sección es si la perspectiva o enfoque de género se ha convertido en eje transversal de la política de la Central. Para ello, se abordarán las entrevistas realizadas a Mabel Gabarra y Estela Díaz, así como también se analizan los principales documentos elaborados por la CTA para elaboración o implementación de las políticas más significativas a lo largo de su historia.

Retomando los testimonios de las entrevistadas y otras fuentes bibliográficas, se pueden señalar algunos hitos que marcaron el devenir de la CTA, entendiendo "la capacidad que esos acontecimientos han tenido para desencadenar situaciones y decisiones posteriores (...) se descubren a través de las consecuencias que tuvieron en su momento, de la huella que dejaron bien a nivel individual y o colectivo" (Tarducci y Rifkin, 2010, p. 17).

En este sentido, es importante tener en cuenta la fundación del CTA en el año 1992, la Marcha Federal en 1994, la constitución del FRENAPO en el 2000, el Movimiento Político, Social y Cultural de 2002 y la Constituyente Social lanzada en 2008, y para cada uno de estos momentos e iniciativas se seleccionó un documento ${ }^{16}$ que contuviese los elementos principales de la propuesta política.

Luego de su análisis, se observa que no se hace mención a los conceptos propuestos en este trabajo, no se menciona al movimiento de mujeres y feminista y se destaca el lenguaje sexista con que se expresan, utilizando el masculino como categoría universal. La única excepción es "Hacia una Constituyente Social", documento que promueve esa iniciativa, en donde menciona a la "militancia de género", entre muchas otras. De todas formas, se resalta un informe elaborado por la Secretaría de Género para marzo de 2009 que hace una lectura de coyuntura mencionando los impactos diferenciales entre varones y mujeres, en tanto aporte a la Constituyente Social, así como también poniendo la agenda feminista y las demandas en el desarrollo del mismo (Secretaría de Género CTA, 2009).

Asimismo, previamente a la constitución de la Secretaría de Género, existen tres elementos para mencionar que comprometen a la Central en su conjunto: en primer lugar, la afiliación

\footnotetext{
${ }^{16}$ Nos referimos a los documentos consultados en formato papel, disponible en el archivo de la sede nacional de CTA. Los mismos no han sido digitalizados por el momento y su autoría corresponde a la CTA. Estos son: Declaración de Burzaco, del 17 de diciembre de 1991; Volante convocando a la Marcha Federal de 1994; Fundamentos, propuestas y estrategia del Movimiento por la Consulta Popular, de marzo de 2001; Documento para el debate 1: apuntes sobre nuestra estrategia, CTA, de septiembre de 2002; y Hacia una Constituyente Social en la Argentina, elaborado en marzo de 2007.
} 
individual, que permitió la inclusión de activistas feministas a esta herramienta; en segundo término, la incorporación de nuevos sujetos organizados en agrupamientos gremiales no tradicionales, que posibilitó el ingreso de AMMAR a la CTA y; por último, la votación a favor de la despenalización del aborto en el Congreso Nacional de la CTA del año 1999, en palabras de Mabel Gabarra ${ }^{17}$ :

En el '99 cuando fue el Congreso que se crea la Secretaría (de género), este grupo de mujeres impulsó en ese congreso la definición de la CTA por la legalización del aborto y se aprobó por aclamación. Pero en ese momento no había una articulación de la lucha por el derecho al aborto, entonces no había una participación expresa, recién se da esta participación con la Campaña (por el derecho al aborto legal, seguro y gratuito) en el 2005 (Mabel Gabarra, entrevista personal, 20 de mayo, 2017).

En la declaración final de este Congreso (CTA, 1999), aparece la mención a las mujeres y las compañeras, aunque no se utilizan categorías como género, segregación horizontal y vertical, movimiento de mujeres y feminista para analizar la coyuntura social, política y económica.

En el año 2000, con la reforma del estatuto, se incorporó la Secretaría de Género e Igualdad de Oportunidades que fue un punto de inflexión para la institucionalización de los espacios de mujeres y de la perspectiva de género. Dicha Secretaría tenía como funciones:

a) elaborar las políticas de la Central que tiendan a igualar los derechos de hombres y mujeres trabajadoras,

b) generar programas de formación para mujeres,

c) generar programas tendientes a la equiparación e igualdad de oportunidades entre hombres y mujeres,

d) representar a la Central en todos los organismos, congresos y/o eventos que tiendan a reivindicar la igualdad de género y los derechos de la mujer,

e) elaborar propuestas de lucha contra toda discriminación y particularmente la de género. En todos los casos, para la Secretaría de Género e Igualdad de Oportunidades deberá nombrarse en ese cargo a una mujer.

\footnotetext{
${ }^{17}$ Mabel Gabarra es abogada, integrante de la Campaña Nacional por el Derecho al Aborto Legal, Seguro y Gratuito, y feminista. Fue integrante del Instituto de Estudios Legales y Sociales (INDESO-Mujer), ONG surgida en 1984 que brindaba asesoramiento jurídico en cuestiones de violencia, trabajo y familia. Fue parte de las Comisiones Organizadoras de los Encuentros Nacionales de Mujeres llevadas a cabo en la ciudad de Rosario.
} 
La primera en asumir esta secretaría fue Mabel Gabarra, que no provenía de ningún sindicato ni organización perteneciente a la CTA, y fue convocada por Víctor De Gennaro debido a su trayectoria en el feminismo, tal como lo expresa a continuación:

A mí me parecía un desafío bastante interesante, yo le dije voy a entrar, voy a estar en la lista, lo que voy a hacer es poner en marcha la Secretaría, organizarla, ver lo que se puede hacer, pero yo no voy a seguir en ese lugar porque, primero, no soy sindicalista, no pertenezco a ningún sindicato y es importante pertenecer a algún sindicato, cuando vos estás en esas instancias. Dentro de tres años vamos a promocionar compañeras (cuando haya nuevamente elecciones) que tomen esto. Esa fue mi idea. Fue mucho sacrificio para mí, porque yo iba a buenos aires una vez por semana, el hecho de no pertenecer a ningún sindicato, nadie me bancaba, los gastos me los bancaba yo, no tenía presupuesto. O sea, cuando empecé a ir era una desolación total y las feministas de buenos aires se sintieron excluidas por el hecho de que Víctor De Gennaro me vino a buscar a mí, como yo no sabía entre mal parada con ese grupo (Mabel Gabarra, entrevista personal, 20 de mayo, 2017).

Su principal tarea en estos años fue organizar las secretarías de género en todo el país y los encuentros nacionales entre todas ellas para definir agendas en común:

Me la pasé viajando en ómnibus, al norte, al sur, era la primera vez que se armaban cosas desde el punto de vista de las mujeres, entonces se armaron encuentros regionales, esto fue entre el 2000 y el 2003. Y yo cuando me voy en el 2003 dejé armado un grupo en cada lugar, de gente que todavía ahora está militando, que siguió ocupando los lugares (...) Esta experiencia a nivel de la gente fue riquísima, muchas compañeras empezaron a tener un proceso, a tomar conciencia de las dificultades con respecto a la actividad sindical, de lo invisibilizadas que estaban en sus gremios, de qué pasaba a nivel nacional con la representación sindical y las mujeres, cómo eran los sindicatos de verticalistas. Eso fue un buen proceso, pero la cuestión fue que fue muy corto lo mío, yo no te puedo hacer una evaluación, después tomó otro cariz y hoy tiene una participación mucho más grande mujeres (Mabel Gabarra, entrevista personal, 20 de mayo, 2017).

A la vez que se empezaban a consolidar las secretarías de género y los grupos de mujeres, de manera artesanal y sin presupuesto asignado, se ponía en juego otra estrategia vinculada a lograr que la perspectiva de género no se redujera a los ámbitos antes mencionados, sino que atravesara al conjunto de la institución. 
Estela Díaz (a cargo de esta secretaría para el período 2003-2006) y Alejandra Angriman (que ocupó el cargo entre los años 2006-2009) ${ }^{18}$ plantean que se hicieron esfuerzos por no quedar encapsuladas en los espacios de mujeres y en incorporar este enfoque en campañas importantes de la CTA como "El hambre es un crimen"; sin embargo, estos intentos no han tenido la efectividad esperada. En la práctica, concluye Estela, esta cuestión sigue siendo un tema de importancia solo para algunas mujeres (Godinho Delgado, 2009), y relata:

Hay muchas dificultades, las decisiones y las vocerías públicas siguen siendo mayoritariamente masculinas. Las dificultadas son las de todas las organizaciones en el patriarcado, con un plus creo en el mundo sindical. Todavía hay una cultura de lo sindical y lo laboral, construida desde un supuesto trabajador ideal varón, la negociación sindical parece definirse desde valores que se asignan a la masculinidad: fuerza, firmeza, prepotencia, poder. Se vienen haciendo acciones para modificar, hay más presencia de mujeres, también de muchas jóvenes, pero esto sigue siendo una tarea que encaramos fundamentalmente las mujeres. Con algunos aliados, pero siempre hay que remarla para lograr que se coloquen mujeres en los actos, que hablen, ni que hablar en las mesas chicas de decisión, que suelen ser casi exclusivamente masculinas. También hay mucho debate respecto al lenguaje no sexista. Son batallas que se van dando y en parte se vienen ganando, con avances y retrocesos (Estela Díaz, entrevista personal, 06 de junio, 2017).

Sin embargo, la incorporación de una agenda con cuestiones destinadas a modificar algunos de los condicionantes estructurales para la participación efectiva de las mujeres en la vida sindical fue adquiriendo mayor centralidad.

En los últimos años crece la preocupación por la inclusión de temas de la agenda feminista como la cuestión de las licencias parentales, las políticas de cuidado, las licencias por violencia de género, entre otros. Estas demandas pueden verificarse también en los sindicatos integrantes de la CTA, que han profundizado la inclusión de una agenda de género, especialmente, a partir de la irrupción del acontecimiento Ni una Menos, del 3 de junio de 2015.

A partir de aquí, es posible concluir que, si bien se produjeron numerosos avances en las reformas de los estatutos como en las resoluciones de los congresos, los documentos escritos para la elaboración e implementación de políticas no promueven la participación de las mujeres en la organización ni la transversalización de la perspectiva de género. No obstante, como se ha podido observar, la agenda de "temas de género" fue incrementándose en relación con las demandas sectoriales para incluirlos.

\footnotetext{
${ }^{18}$ En la actualidad, Alejandra Angriman y Estela Díaz continúan a cargo de la Secretaría de Género en la CTA Autónoma y la CTA de los trabajadores, respectivamente.
} 


\section{Instancias de articulación entre la CTA y el movimiento de mujeres y feminista}

En este apartado se indican y describen las instancias de articulación entre la central y el movimiento de mujeres y feminista. Partiendo de la pregunta central que orienta este trabajo sobre la presencia de las mujeres como sujetos políticos, se identifican los espacios de articulación política en esos 19 años. Para ello se hace énfasis en tres espacios: los Encuentros Nacionales de Mujeres, la Campaña Nacional por el Derecho al Aborto Legal, Seguro y Gratuito, y la Marcha Mundial de Mujeres. Y se recuperan las entrevistas realizadas para este trabajo, artículos periodísticos, sitios oficiales de las organizaciones mencionadas y de la CTA, así como documentos elaborados por la Secretaría de Género e Igualdad de Oportunidades. En este sentido es importante destacar el rol fundamental que ha ejercido la Secretaría de Género desde sus inicios:

Siempre la Secretaría ha tenido una fuerte articulación con todas las organizaciones integrantes de la CTA, además de una articulación más general con el movimiento de mujeres, así como movimientos políticos y sociales (Estela Díaz, entrevista personal, 06 de junio, 2017).

A nivel global se desarrolló la Marcha Mundial de Mujeres (en adelante MMM) que es una articulación internacional de acciones feministas que integra a organizaciones y grupos que trabajan para combatir la pobreza y todas las formas de violencia hacia las mujeres. Tiene como propósito luchar contra toda forma de discriminación y desigualdad vivida por las mujeres, propiciando el cambio social, político y económico y la vinculación entre el movimiento de mujeres y otros movimientos sociales progresistas (Marcha Mundial de Mujeres, s.f.).

Sus antecedentes se encuentran en la Marcha de las Mujeres en Quebeq en 1995, en la cual 850 mujeres marcharon durante diez días para llevar nueve reivindicaciones económicas. Esta articulación internacional ganó una importante adhesión de la población y el movimiento feminista, y, a su vez, aportó la idea de mundializar estas acciones. Sin embargo, fue en el Foro Mundial de Beijing, en donde se realizó esta convocatoria. El ocho de marzo de 2000 se lanzó oficialmente la MMM a través de una conferencia de prensa en las ciudades de Montreal, Nueva York y Ginebra. Dicha organización está presente en 163 países y congrega a más de 5.000 grupos; sus acciones son de formación y movilización en cuatro campos: autonomía económica de las mujeres, violencias contra las mujeres, paz y desmilitarización, y bien común y servicios públicos (Pascielli, 2015).

La CTA es integrante de esta articulación en el capítulo argentino, en conjunto con un gran número de agrupamientos, y participó de las acciones definidas por la MMM en estos campos, así como de sus posicionamientos.

En el plano nacional, se encuentra la Campaña Nacional por el Derecho al Aborto legal, seguro y gratuito que tiene como antecedentes más directos a la "Asamblea por el Derecho al Aborto", surgida en la Ciudad de Buenos Aires en el año 2002 al calor de la participación 
popular en distintas asambleas barriales; el ENM de 2003 en la ciudad de Rosario, en donde se realizó una asamblea por el derecho al aborto al final de la primera jornada de talleres, y se discutieron estrategias y acciones nacionales para conseguir su legalización; también aparecen allí, por primera vez, los pañuelos verdes que tiñeron la multitudinaria marcha del día domingo, distribuidos por un grupo de católicas por el derecho a decidir (Alma y Lorenzo, 2009); y el ENM de la ciudad de Mendoza en 2004.

Esta articulación fue impulsada por un conjunto de militantes feministas, grupos de mujeres y militantes pertenecientes a organizaciones políticas y sociales, y se lanzó el 28 de mayo de 2005. La integran centenares de colectivos y personalidades vinculados a los derechos humanos, a ámbitos académicos, sindicatos, movimientos sociales y culturales, comunicadoras, etc. Dicha articulación tiene como propósitos principales poner en agenda pública el debate en torna a la despenalización y legalización del aborto, acercar cada vez más espacios a esta demanda y la aprobación de la normativa que garantice este derecho; su principal lema es "Educación sexual para decidir, anticonceptivos para no abortar, aborto legal para no morir" (Aborto legal, s.f.).

La CTA participa de esta articulación desde sus orígenes, e incluso ya se había acordado su postura sobre el tema en un congreso previo; sin embargo, el trabajo efectivo sobre la cuestión tuvo mayores dificultades, como bien nos cuenta Mabel:

Hubo una marcha el 25 de noviembre de 2005 donde se entregaron las firmas al Congreso Nacional donde la CTA se manifestó más abiertamente que estaba a favor del aborto, pero siempre con resistencias al interior. Es decir, se tomaban definiciones así, pero a la hora de trabajarlas hacia adentro encontrábamos dificultades, no sólo con las direcciones sino con las mujeres. El tema del aborto es un tema muy complejo y cuesta, a veces, tratarlo cuando hay mucha gente católica, que por cuestiones religiosas no lo acepta. Es decir, se podían trabajar un montón de temas con las mujeres, pero ese era el más complejo (Mabel Gabarra, entrevista personal, 20 de mayo de 2017).

También, a nivel local, emergen los Encuentros Nacionales de Mujeres (en adelante ENM) que empezaron a desarrollarse en el año 1986, en diferentes ciudades de Argentina. Dichos encuentros son señalados como antecedentes directos los Encuentros Feministas Latinoamericanos y del Caribe, que se celebran desde comienzos de la década de 1980. Asimismo, en el año 1985, surge el "Encuentro Internacional de Mujeres" organizado por Naciones Unidas en Kenia. Los ENM se realizan una vez al año, duran tres días consecutivos, y se han sostenido de manera ininterrumpida hasta la actualidad, aumentando año a año la cantidad de mujeres que congrega. Sus principios son la autogestión, la autonomía, la horizontalidad, la democracia, la autoconvocatoria y el autofinanciamiento (Alma y Lorenzo, 2009). 
Los ENM son una instancia fundamental para la práctica feminista de encontrase, hablar, intercambiar experiencias y construir un registro político de las distintas opresiones que sufren las mujeres. Se genera "empoderamiento" en la pertenencia a una identidad colectiva que posibilita la construcción de autonomía y la valorización propia en cada ámbito cotidiano, así como también es un espacio en el que se elaboran estrategias para la concreción de las demandas que tiene el movimiento. Las mujeres de la CTA participan de estos encuentros desde su fundación, al respecto, Estela Díaz cuenta como comenzó esto:

Desde el nacimiento la CTA está articulada con el movimiento de mujeres, como también lo hace con otros movimientos sociales. En los primeros congresos ya se comenzó a definir un programa político-sindical que abarcaba temas como la violencia de género y los derechos sexuales y reproductivos. En el $3^{\circ}$ congreso se adhiere al reclamo por despenalización del aborto. Participamos desde el nacimiento de la CTA en los encuentros nacionales de mujeres y estuvimos en la reunión inaugural que definió la creación de la Campaña Nacional por el Derecho al Aborto legal, seguro y gratuito en el año 2005 en Córdoba (Estela Díaz, entrevista personal, 06 de junio de 2017).

También Mabel Gabarra se refiere a esto cuando señala que:

Nosotras en los tres años (2000/2003) participamos en los Encuentros Nacionales de Mujeres, organizábamos encuentros previos y había muchas mujeres que participaban (...) Las mujeres participando en el movimiento de mujeres y feminista empiezan a tener mayor referencia pública y eso fuerza a que sean más reconocidas en sus propias organizaciones. Las eleva en su importancia política (Mabel Gabarra, entrevista personal, 20 de mayo de 2017).

Desde sus orígenes la CTA ha sido parte de diversas articulaciones con el movimiento de mujeres, así como ha incorporado a activistas feministas a su estructura, recuperando su agenda y calendario de acciones y también construyendo referencias públicas en la articulación de ambos movimientos, convirtiéndose en un elemento fundamental.

\section{A modo de cierre}

Hasta aquí se procuró revisar el proceso de participación femenina en instancias de conducción de la CTA, desde sus orígenes hasta su ruptura revisando la trasnversalización de la perspectiva de género y aspectos vinculados a la articulación entre la central sindical y el movimiento de mujeres y feminista.

Con esta aproximación se ha procurado dar cuenta de aspectos invisibles en la política sindical y en la historia de la CTA. Las dimensiones analizadas se basan en una mirada crítica que recoge aportes de los estudios de género y aborda el punto de vista o la perspectiva de algunas de sus protagonistas. En este recorrido se han detectado otras 
dimensiones vinculadas con las condiciones de trabajo, los crecientes niveles de precarización y flexibilización laboral, la feminización de la pobreza, la agenda feminista en el campo de los conflictos sindicales contemporáneos, que requieren nuevas inmersiones investigativas para profundizar la comprensión del fenómeno estudiado.

Somos conscientes de que abordar estas dimensiones se torna crucial porque están atravesadas por problemas estructurales y dificultades cotidianas que tienen su origen en la naturaleza jerárquica y sexista de las relaciones laborales con impacto directo en la vida de las personas trabajadoras, incluyendo las experiencias sindicales con sus lógicas de organización, estilos de conducción y modos de funcionamiento androcéntrico.

Del análisis global aquí presentado - basado en las entrevistas realizadas y los documentos consultados- se desprende que la experiencia de la CTA fue un tipo de respuesta a las transformaciones políticas, económicas y sociales sufridas en Argentina a partir de los impactos generados por la reconversión neoliberal; y fue también un intento de construcción de un modelo sindical alternativo al de la CGT, con el acento puesto en colectivos sociales emergentes e incorporando, paulatinamente, las causas impulsadas por los movimientos feministas.

Desde un inicio la articulación entre la central y el movimiento de mujeres estuvo presente como un eje político que atravesó la experiencia y fue uno de los posibilitadores de la existencia de espacios específicos en donde las mujeres pudieran organizarse, más allá de no contar con la legitimidad dada por la institucionalidad de los mismos; también generó la apropiación de la agenda y los debates y demandas del movimiento por parte de las sindicalistas involucradas en estas cuestiones.

Estos corrimientos de sentidos y propuestas políticas, no tuvieron un correlato directo en dotar de centralidad a la participación de las mujeres y otras identidades feminizadas en los ámbitos de conducción. A partir de este trabajo se ha podido constatar que la participación de las mujeres en la instancia de conducción nacional de la CTA fue muy marginal y solo alcanzó el 30\% establecido en las elecciones de año 2006, a partir de la normativa específica que indicaba su representación por cupo como medida afirmativa.

Esta tendencia se ha podido precisar observando que, a excepción de un solo caso, los cargos ejercidos por mujeres no se extendieron por más de un período y su mayor crecimiento se produjo en secretarías que tienen a su cargo la formación, comunicación y acción social, funciones asociadas a características femeninas. Tales elementos son expresiones de la división sexual del trabajo y las relaciones de poder entre varones y mujeres e indica que, si bien la política de cuotas favoreció la participación de una "masa crítica" para atravesar el techo de cristal, también operaron estereotipos que distribuyeron a las mujeres en determinados roles. 
Los argumentos a favor de las medidas de acción positiva para promover la mayor participación femenina en la dirigencia sindical se basaron, principalmente, en la idea de "minoría crítica", para que las mujeres puedan ocupar lugares dentro de las organizaciones gremiales con el fin de incluir en los modos de ejercicio de poder y en la inclusión de temas específicos en la agenda sindical (Aspiazu, 2015).

Asimismo, los principales condicionantes que hicieron que estas mujeres no continúen en las secretarías que tuvieron a su cargo, se vinculan al androcentrismo presente en los espacios de toma de decisiones, elementos expulsivos para las sindicalistas que ingresan a los mismos. Además, las exigencias de un tiempo intensivo para la participación política de las mujeres, implicó dificultades concretas para conciliar la vida familiar, laboral y gremial, impactando negativamente en la calidad de vida y la posibilidad de sostener una permanencia constante en cargos y funciones.

Por último, la transversalización del enfoque de género como mecanismo propuesto para permear toda la organización sindical fue deficitaria y no alcanzó un impacto significativo, a pesar de que la institucionalización de la Secretaría de Género e Igualdad de Oportunidades, la mayor participación de mujeres en la vida sindical y en los ámbitos de decisión y el crecimiento del movimiento de mujeres y feminista, pueden interpretarse como elementos fundamentales para avanzar hacia esos cambios.

En síntesis, el proceso de participación de las mujeres en la CTA y de inclusión del enfoque de género, si bien tuvo algunos avances a lo largo de esos años, es complejo y reconoce potencialidades y limitaciones a la hora de analizarlos. El desafío se encuentra también en el desarrollo de nuevas generaciones de dirigentes formadas al calor de las transformaciones producidas por los feminismos en nuestro país y en el mundo. De ello se desprende la necesidad de dar continuidad a las líneas de investigación que se abren para profundizar su desarrollo en el futuro, indagando los impactos que el acontecimiento "ni una menos" ha producido desde la primera movilización masiva, realizada el 3 de junio de 2015 en el ámbito sindical, en general, y en la CTA, en particular, entre otros acontecimientos relevantes.

\section{Referencias bibliográficas}

Aborto legal. (s.f.). Campaña Nacional por el Derecho al Aborto Legal, Seguro y Gratuito. Recuperado de http://www.abortolegal.com.ar/about/

Alma, A., y Lorenzo, P. (2009). Mujeres que se encuentran: una recuperación histórica de los Encuentros Nacionales de Mujeres en Argentina,
1986-2005. Buenos Aires: Feminaria Editora.

Armelino, M. (2004). La protesta laboral en los años 1990. El caso de la CTA. Estudios del trabajo, (28), 3-27.

Aspiazu, E. (2014). Equidad de género, mercado de trabajo y sindicalismo en 
la Argentina. Realidad Económica, (284), 10-36.

Aspiazu, E. (2015). Participación de las mujeres e institucionalidad de la problemática de género en el sindicalismo argentino. $12^{\circ}$ Congreso Nacional de Estudios del Trabajo. Organizado por Asociación Argentina de Especialistas en Estudios del Trabajo (ASET), Buenos Aires. Recuperado de http://nulan.mdp.edu.ar/2685/1/aspia zu-2016.pdf.

Basualdo, E. (2003). Las reformas estructurales y el Plan de Convertibilidad durante la década de los noventa. Realidad Económica, (200), 42-83.

Bonaccorsi, N., y Carrario, M. (2012). Participación de las mujeres en el mundo sindical: Un cambio cultural en el nuevo siglo. La Aljaba, (16), 125-140.

Burton, J. (2013). Aproximaciones al movimiento de mujeres y al feminismo en Argentina, 1970 -post 2001. X Jornadas de Sociología. Organizado por la Facultad de Ciencias Sociales. Buenos Aires.

Central de Trabajadores Argentinos. (1999). Documento del 2do Congreso Nacional de Delegados de la CTA. Recuperado de http://catalogo.cedinci.org/cgibin/koha/opacdetail.pl?biblionumber $=36506 \&$ shelf browse_itemnumber $=34440$.

Castellani, A., y Schorr M., (2003). ¿Crisis? ¿Qué crisis? Notas sobre la debacle del régimen convertible en la Argentina. III Jornadas de Sociología de la UNLP. Organizado por Departamento de Sociología de la Facultad de Humanidades y Ciencias de la Educación. La Plata.
Cortés Conde, R., (2003). La crisis argentina de 2001-2002. Cuadernos de economía, (40), 762-767.

Cutuli, R., y Martino, A. (2012). Políticas públicas de conciliación entre trabajo y cuidado: propuestas para su conceptualización a partir del caso argentino. Estado, políticas sociales y sociedad. Tandil: Debates latinoamericanos.

Eagly, A., y Carli, L., (2007). Through the labyrinth: The truth about how women become leaders. Boston: Harvard Business Press.

Gerez, M. J. (2018). Feminizar la política es lo que va a salvarla. En V. Freire (Ed.) La cuarta ola feminista. Buenos Aires: Ulises Bosia.

Godinho-Delgado, D. (2009). Sindicalismo latinoamericano y política de género. Montevideo: $\quad$ Friedrich-EbertStiftung.

Gusmerotti, L. (2009). La influencia de la tradición histórica en la configuración de la identidad social y política de la Central de los Trabajadores Argentinos (CTA). Cuadernos de H ideas (3), 22-39.

Hirata, H. (1997). Contribución a la discusión sobre el concepto de trabajo. En H. Hirata y D. Kergoat (Ed.), La división sexual del trabajo. Permanencia y cambio (pp. 53-64). Buenos Aires: Asociación Trabajo y Sociedad.

López-Diez, P. (2002). El techo de cristal también existe en los medios. En Mujeres, hombres y medios de comunicación (pp. 291-300). Valladolid: Lex Nova: Junta de Castilla y León, Dirección General de la Mujer e Igualdad de Oportunidades.

Marcha Mundial de Mujeres. (s.f.). Objetivos de la marcha mundial. Recuperado https://marchemondiale.org/index.ph 
p/quienes-somos/objetivos-de-lamarcha-mundial/?lang=es.

Pascielli, A. (2015). Marcha Mundial de Mujeres capítulo Argentina. Marcha. Una mirada popular y feminista de la Argentina y el mundo. Recuperado de:

http://www.marcha.org.ar/marchamundial-de-mujeres-capituloargentina-24-horas-de-accionfeminista/.

Pateman, C. (1995). El contrato sexual. Barcelona: Anthropos Editorial.

Pautassi, L. (1998). ¿Primero... las damas? La situación de la mujer frente a la propuesta del ingreso ciudadano. Estudos feministas, (18), 46-70.

Presidencia de la Nación, Ministerio de Trabajo, Empleo y Seguridad Social. (s.f.). Las mujeres en el mundo del trabajo. Argentina: Presidencia de la Nación, Ministerio de trabajo, empleo y seguridad social. Recuperado de: https://www.argentina.gob.ar/sites/de fault/files/informe_ctio_documentod etrabajo.pdf.

Retamozo, M., y Morris, M. B. (2015). Sindicalismo y política. La Central de Trabajadores de la Argentina en tiempos kirchneristas. Estudios sociológicos, (97), pp. 63-87.

Rodríguez-Enríquez, C. (2015). Economía feminista y economía del cuidado. Aportes conceptuales para el estudio de la desigualdad. Nueva sociedad, (256), 30-44.

Rojo-Brizuela, S., y Tumini, L. (2008). Inequidades de género en el mercado de trabajo de la Argentina: las brechas salariales. Revista de Trabajo, (6), 53-70.

Secretaría de Género CTA. (2009). Informe Noveno Congreso Nacional de delegados. 30 y 31 de marzo de 2009.
Senado y Cámara de Diputados de la Nación Argentina. (2002). Ley No 25.674 de cupo femenino sindical. Boletín Oficial, 11 de noviembre de 2002.

Sénen-González, C. (2013). La revitalización sindical en Argentina: alcances y perspectivas. Buenos Aires: Universidad Nacional de La Matanza, Prometeo.

Strada, J. (2018). Trabajo no remunerado, segregación ocupacional y políticas de ajuste: los ejes ausentes en el proyecto oficial de Equidad de Género. Centro de Economía Política Argentina. Recuperado de http://centrocepa.com.ar/informes/10 8-trabajo-no-remuneradosegregacion-ocupacional-y-politicasde-ajuste-los-ejes-ausentes-en-elproyecto-oficial-de-equidad-degenero.html.

Tarducci, M., y Rifkin, D. (2010). Fragmentos de historia del feminismo en Argentina. En S. Santoro y S. Chaher (Ed.), Las palabras tienen sexo II. Herramientas para un periodismo de género (pp. 17-39). Buenos Aires: Artemisa Comunicación Ediciones.

\section{Fuentes primarias}

Mabel Gabarra, entrevista personal, 20 de mayo de 2017.

Estela Díaz, entrevista personal, 06 de junio de 2017. 